\title{
Characterising Confounding Effects in Music Classification Experiments through Interventions
}

\author{
Supplementary Material
}

\author{
First Author1, Second Author, and Third Author
}

\section{S1 - Regulated Bootstrap Sampling Example}

To illustrate the resampling strategy described in Alg. 1, consider a collection $D$ with 5 instances per class. We aim to create a pair $\left(D_{t}, D_{p}\right)$ from which to derive a $D_{p}^{\prime}$ with $n_{r}=2$ instances per class. For a given class a $\in A$, assume $D$ contains $\left\{\left(r_{1}, \mathrm{a},\{“ \mathrm{E} "\}\right),\left(r_{2}, \mathrm{a},\{“ \mathrm{E} "\right.\right.$, "F" $\left.\left.\}\right),\left(r_{3}, \mathrm{a},\{“ \mathrm{~F} "\}\right),\left(r_{4}, \mathrm{a},\{“ \mathrm{G} "\}\right),\left(r_{5}, \mathrm{a},\{" \mathrm{H} "\}\right)\right\} . \mathrm{We}$ therefore have $Z=\left\{\right.$ " $E^{\prime \prime}$, " $F$ ", " $G$ ", " $\left.H^{\prime \prime}\right\}$, with some instances sharing values of $z$ and one that takes two. For simplicity, we refer to each instance by its index (i.e., 1, 2, 3, 4, 5).

i) Assume the bootstrap sampling in Phase 1 creates the pair $d_{t}=\{1,3,3,4,5\}$, $d_{p}=\{2\}$. Since the size of the test collection is smaller than $n_{r}$, failing the check in Phase 2, we proceed to curated sampling in Phase 2.

ii) Assume we draw $z=$ "F" and thus we hold out instances $d_{h}=\{2,3\}$. As the size of the hold-out collection matches $n_{r}$, we create a train/test pair avoiding instances in $d_{h}$ for training, such as $d_{t}=\{1,1,4,5,5\}, d_{p}=$ $\{2,3\}$.

iii) Even though $d_{p}$ contains 2 instances, only one of them (3) is regulated, as the other (2) shares value of $z$ ("E") with an instance in $d_{t}(1)$. Therefore, the check in Phase 2 fails, so we attempt curated sampling in Phase 3 again.

iv) Assume we now draw $\mathbf{z}=$ "G", thus holding out instance $4\left(d_{h}=\{4\}\right)$. As the hold-out collection includes only one instance, we keep drawing from $Z$.

v) Assume we draw $\mathbf{z}=$ "H" and thus we append instance 5 to the hold-out collection $\left(d_{h}=\{4,5\}\right)$.

vi) Since $\left|d_{h}\right|=n_{r}=2$, we create a new train/test pair avoiding instances in $d_{h}$ for training, such as $d_{t}=\{1,1,3,3,3\}, d_{p}=\{2,4,5\}$.

vii) The number of regulated instances in the testing collection now meets the threshold, so we accept the pair and proceed to Phase 4.

We would repeat these steps for all other classes in the collection to create $D_{t}$, $D_{p}$ and $D_{p}^{\prime}$ through pruning. 


\section{S2 - Regulated vs. Random Subsetting}

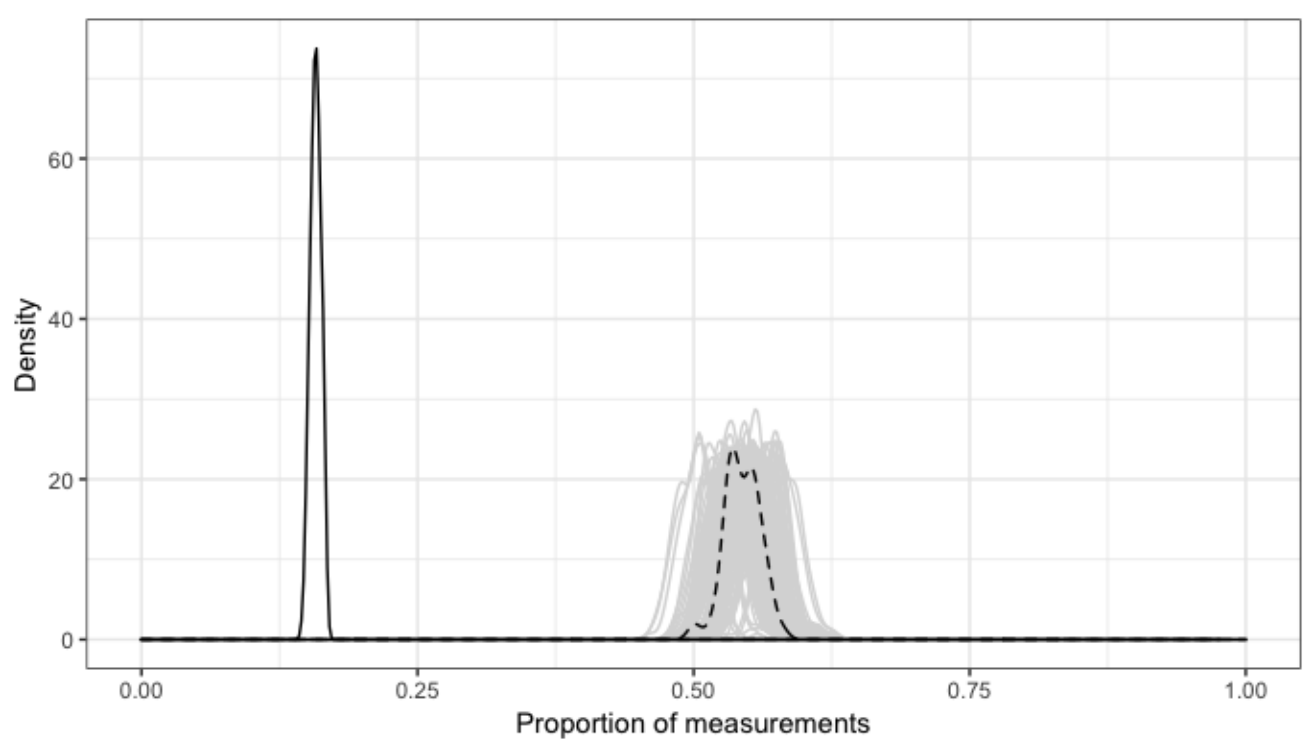

Figure 1: Distribution of the proportion of mean recall measurements larger than or equal to the matching one in a different subset of the test collection with identical number of instances per class, with predictions obtained in original GTZAN recordings. The solid black line represents the measurements in the pruned test sets (i.e., regulated for artist replication) compared with 100 simulations (mean 0.1586, standard deviation 0.0046); the grey lines represent the $100 \times 100$ pairwise comparisons between the simulations, with the dashed black line representing their average (mean 0.544, standard deviation 0.0228). Each simulation comprises 40 randomly sampled sets of instances, one from each of the 40 generated test collections in the reported case study. 


\section{S3 - Interactions between System-Construction Methods and Evaluation Conditions}

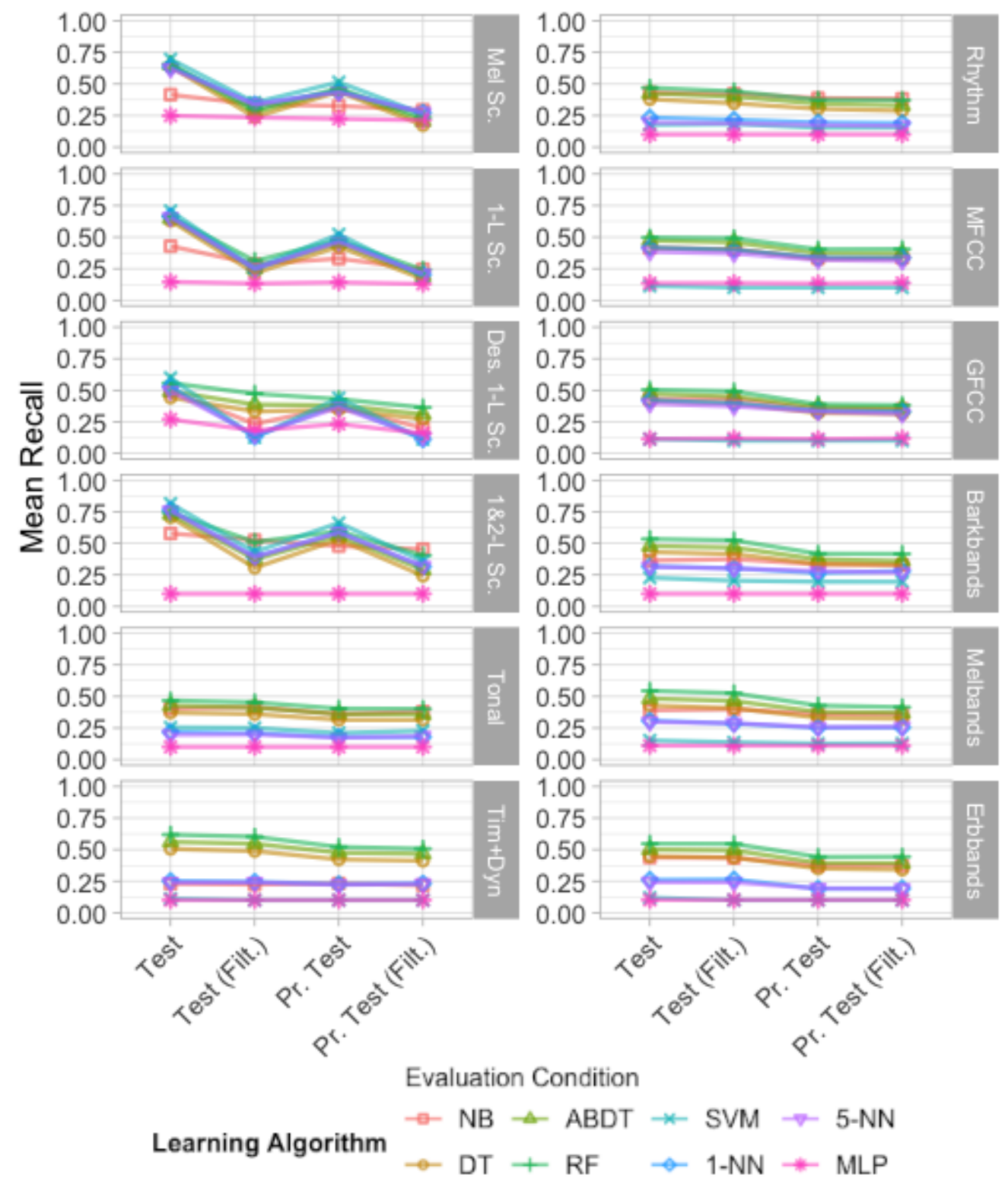

Figure 2: Interaction between learning algorithm and evaluation condition in average mean recall for systems constructed using training collections sampled from $G T Z A N$ with bootstrap regulated over artists across feature sets. 


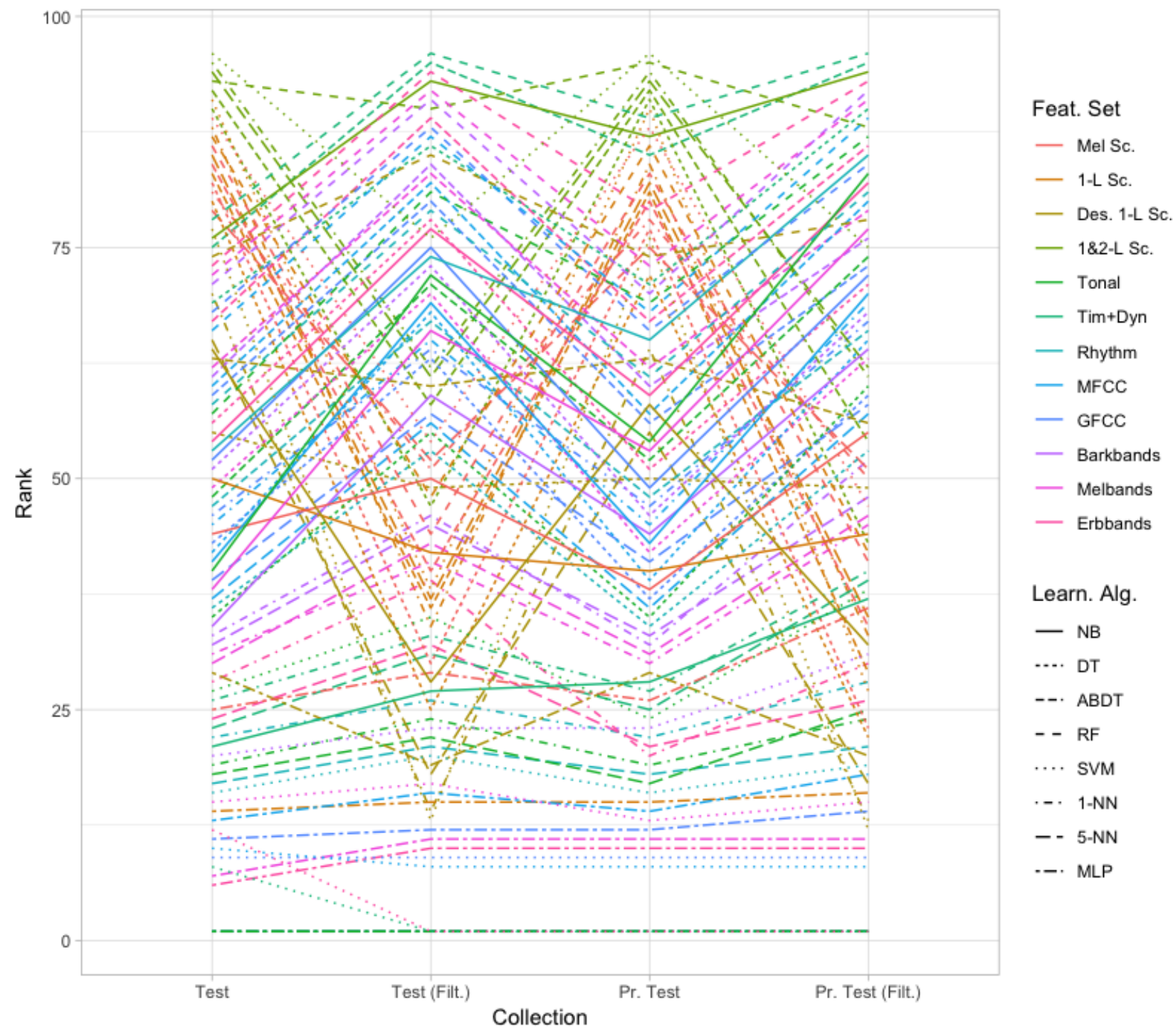

Figure 3: Interaction between system-construction method and evaluation condition in rank of average mean recall for systems constructed using training collections sampled from GTZAN with bootstrap regulated over artists. Ties in average mean recall are resolved by assigning the minimum possible rank to all involved methods. 